\title{
The Design and Application of Cloud Video Conference System
}

\author{
Li Xiaolan \\ Wuhan Vocational College of Software and Engineering, Wuhan, 430205, China
}

Email: 16583752@qq.com

Keywords: cloud video conference system; logic framework design; security system

\begin{abstract}
With the deepening of digital campus construction in colleges and universities, video conference system has become an important part of the construction of campus informatization. It is applied into several different school works including teaching, collaboration and management. To build this system into a more intuitive, timely and efficient remote communication tool, a cloud video conference system featuring "remote, interactive and collaborative" is carried out. The key point of building this system is analyzing the overall planning of cloud video conference platform and overall business logic framework as well as taking the security design system and application security system into consideration.
\end{abstract}

\section{Introduction}

Nowadays, with the rapid development of modern communication network technology, the traditional communication mode cannot meet the people's requirements day by day. [1] The traditional communication in school work mainly depends on telephone contact and centralized meeting which not only delays time but also consumes a lot of resources.[2] With the continuous development of the Internet and the increase of the scale of Internet users, the new media of network video which mainly carries the Internet and mobile Internet has gradually become one of the most widely used network services in China. Video conference, as an intuitive, efficient, timely and low-cost remote communication tool, can effectively solve the problems appeared in daily teaching and management so as to achieve efficient communication within colleges.[3] Video conference can quickly carry out face-to-face visual communication.[4] With the help of video conference, remote meetings and training, remote teaching, remote observation and employment, online streaming media teaching can be arranged at any time.

\section{Advantages of Cloud Video Conference}

Video conference system is authentic, highly efficient and real-time. It is a simple and effective technical means for management, command and collaborative decision-making. It has been widely used in China. With an advanced video conference system, users can get the following benefits:

\subsection{Saving travelling and time expenses for trans-regional meetings}

With respect to many foreign-funded enterprises in Beijing, workers from companies like Motorala, HP, SIEMENS, P\&G, Shell Oil Company, Lucent, NOKIA, Coca-Cola spend high annual business expenses.[5] The time spent on their way to meeting place occupies $80 \%$. It can be seen that applying video system can largely save time and money.

\subsection{Reducing travel expenses}

In this worldwide information age, communication is common everywhere, business travel is inevitable for company workers. But in fact, more than $50 \%$ of communication does not need to be face-to-face.[6] The lack of effective remote communication tools causes the increase of communicating cost. Long-distance presentation? Long-distance negotiation? Long-distance training? These are not available several years ago because of technical constraints and high costs. But after the popularity of video conference renting mode, everything changed. As long as the 
cameras and headphones are ready, you can enter the website into your own conference room. In the network conference room, the realization of various academic activities is easier.

\subsection{Improving the efficiency of meetings}

Since the cost of video conference is roughly proportional to the time of the meeting, it is necessary to save meeting time and improve efficiency. And with the reachable conference materials, documents, physical objects, it is possible enough to fully exchange information with each other.

\subsection{Involving more attendance}

On many occasions, many delegates are unable to attend the meetings because of working stress or limited funding. The use of video services can solve this problem well and attract more people to attend the meeting. In addition, when using video conference to conduct research and make plan, it is convenient to involve extra delegates to the conference at any time so as to collect ideas of more and more people.

\subsection{Providing a wider range of recruitment opportunities}

The application of video conference system gives full play to the essence of network recruitment, which means it is not limited by geography, venue costs and transportation costs, and it is also more time-saving.

\subsection{Expanding cloud video conference platform}

Cloud video conference platform itself relies on the cloud architecture, which can easily and quickly expand its own capacity by adding MCU. When upgrading the platform, it does not need additional development or redeployment. It can achieve seamless docking only by adding MCU or adding MCU from other branches into cloud platform.

\section{The Design of Cloud Video Conference Application System}

\subsection{Overall plan}

The construction of high-definition video conference of this school is based on video information security standards.[7] Supporting by internal network structure of school, this video conference system is devoted to building a comprehensive visual video communication platform. From the overall structure, this project needs to establish a DMZ generator room to deploy the cloud video conference center, including the following servers:

MCU server: provide core support of multipoint video conference;

Cloud video management server: provide user management system, conference room rental system and flow billing system and other application systems;

SIP server: provide SIP service parsing, provide support for voice calls and traditional telephone conferences;

Mobile video access server: manage the mobile terminal devices through the firewall to access the video conference center;

Conference management control server: provide video conference on-site operation platform;

Recording server: providing video conference recording.

Cloud video system is deployed in the DMZ generator room. For the campus network users, they can have access to this system through the existing campus internal network. For the off-campus users, this system can provide services to the external network terminal users through the firewall.

\subsection{General logic framework}

In order to divide the platform framework more clearly, we can start with the logic elements of the system, and divide the construction framework of this project into four levels: access layer, application layer, service layer and resource layer. This is shown in Figure 1: 


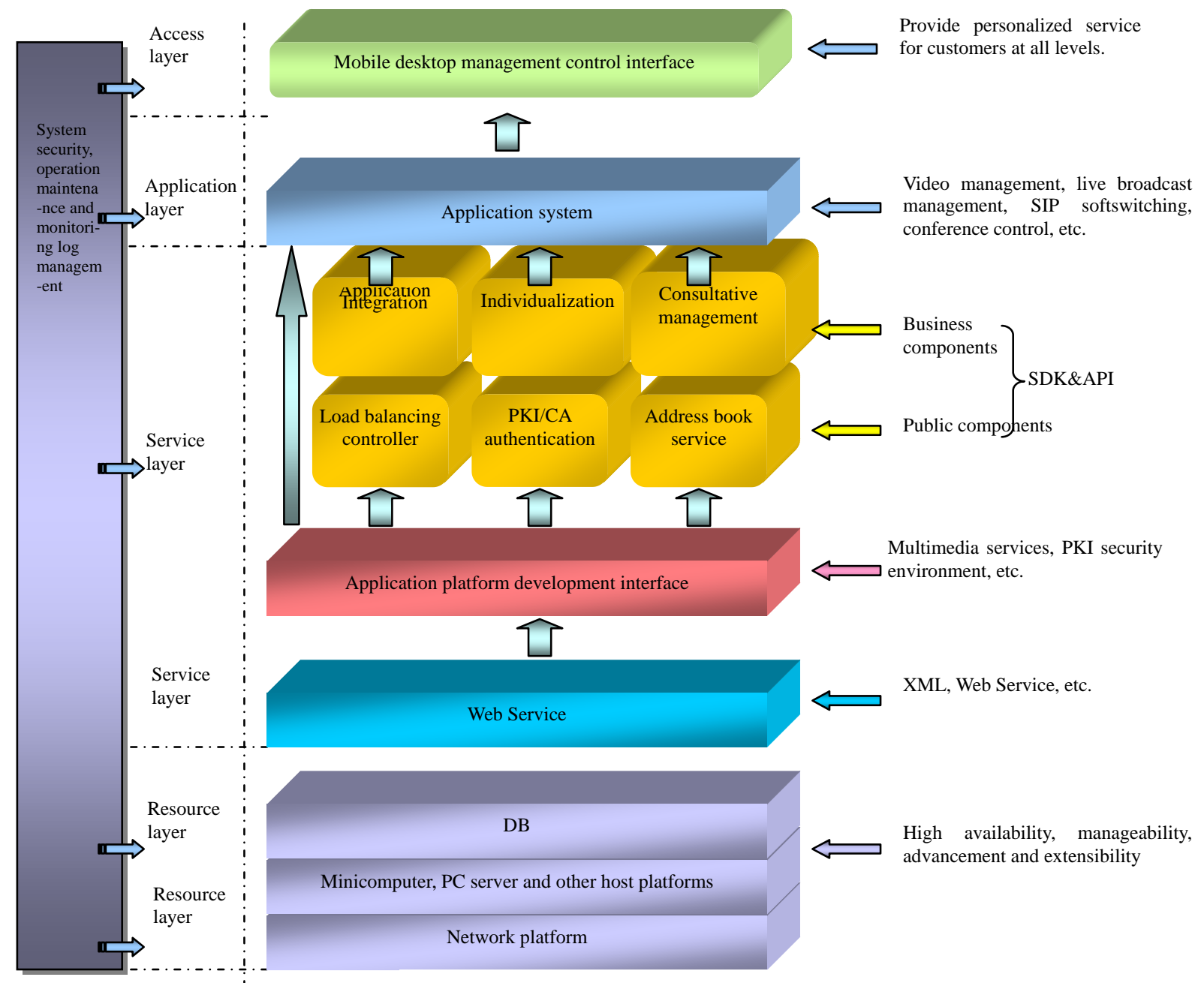

Figure 1 General logic

The general logic framework consists of access layer, application layer, service layer and resource layer from top to bottom. The whole framework embodies as Figure 2: with application layer and service layer as the core, resource layer as the backing, customers at all levels can accept high-quality personalized services through access layer in a variety of ways.

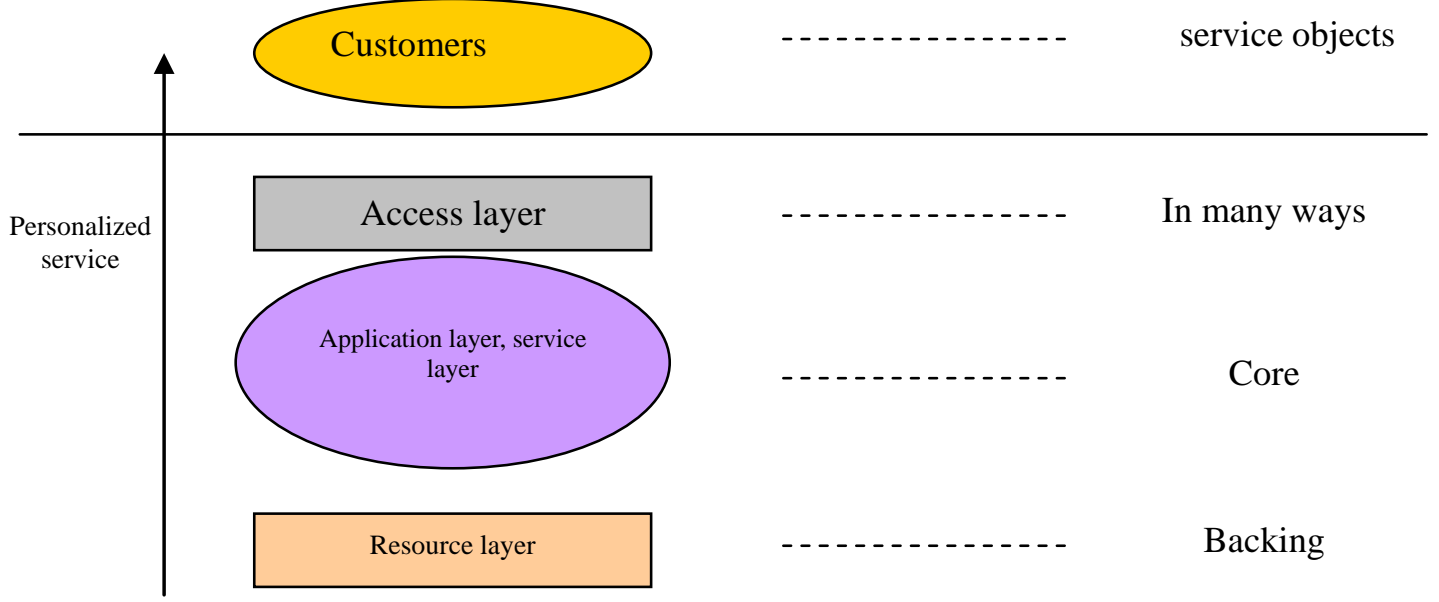

Figure 2 Personalized services

\subsubsection{Access layer}

Users can login information system through Web and get personalized information service. After figuring out the application type and content that users need, the video conference system would 
authenticate users' identity through the unified address book and provide personalized customization for them.

\subsubsection{Application layer}

Application layer is the core of the whole logic framework. By calling the application support platform of the service layer, this layer constructs logic group of applications in the form of templates and components, then provides services for the end users.

\subsubsection{Service layer}

Service layer and application layer together constitute the core of the whole logic framework. The application basic system is composed of templates, business components and public components. It is the software support platform of application layer. Through the service layer, we can quickly create, assemble, deploy, and manage dynamic and robust application logic. The service layer is a based application platform, which is divided into three levels. The bottom two levels are the basic developing platform, which are based on distributed application development environment and development interface of system platform. The other level is the application-based system which provides the core service functions in different application system and works as basic service platform for application logic operation.[8] The application-based system is composed of component-based function packages, templates and secondary development interfaces. It provides stable, safe and simple bottom-level function for the application modules developed and operated on it. It lays a foundation for forming an integrated application as well as guarantees the maintainability and extensibility of the system.

\subsubsection{Resource layer}

The resource layer is the supporting environment of application layer and service layer. Network infrastructure provides resource services such as TCP/IP, directory and security, which are accessible through open and standard interfaces and protocols; host systems and functional server clusters provide resource services for application logic, including databases, HTTP, transaction processing, messages and so on. We should scientifically and reasonably plan the resource level according to the needs of construction development. A unified and complete overall logic framework clearly divides the logical levels of the cloud-based video conference platform in colleges and universities. Each level is relatively independent, thus simplifying the system complexity and ensuring that the system meets the overall construction requirements of the project. From the overall logic framework design, we can see that the entire platform is built on the school network platform. The construction of video conferencing platform is based on network physical security. Through hierarchical management, resource integration and sharing, the school cloud video conference system will be built into a new "one-stop" service platform which allows the management of video conference, communication among colleges and universities, and communication among different colleges in a school.

\section{Design of Application System}

The core application system of cloud video conference platform is the management of SIP and MCU. On this basis, the video conference resources are divided by the management of users and terminals and distributed to the whole campus users. The management application layer of cloud video conference needs to manage terminal equipment and provide statistical analysis of video conferencing traffic. After that, it also needs to provide corresponding reports for school leaders to make decisions.

\section{The Security Design of System Platform}

Protecting the security of network and information is an important part of the construction of cloud video conference system. The project should establish a unified network which contains user 
identity authentication, authorization management, responsibility identification, security monitoring, emergency handling and other security strategies to ensure the security of cloud video platform from both technical and management levels.

\subsection{Threat analysis of system security}

Security system is very important to cloud video conference system. The whole system and even the whole school network should consider security problem from several aspects including physical layer, network layer, data information and management. In order to carry out academic activities, school must apply a series of security authentication technologies, such as identity identification, security authentication, transmission of important data after encryption, automatic backup and so on. The safety products and password products must be certified and licensed by the national security and secrecy department.

The security of our school involves all aspects of the platform. In terms of the 7-level model of network OSI, network security runs through it. In terms of the TCP/IP protocol that Internet actually operates, network security of our school also runs through four levels of its information system.

The threats faced by cloud video conference system can be divided into three types: one is the threat of information in the network, such as the problem of sensitive information decryption; second one is the threat of equipment in the network, mainly reflected as the improper configuration; the third one is the threat of human factors, such as man-made attacks, man-made software "backdoor" and man-made imperfect security and confidentiality management system. The solution of man-made threats mainly rely on the formulation and implementation of the system, for example, being strict with the operating habits of relevant personnel could reduce the threat in this regard.

\subsection{Network security design}

Firewall is used to monitor the security of users inside and outside the network, and prevent hackers from invading. Firewall policy is planned as follows: routing mode is adopted for communication among intranet area, server DMZ area and external network port. The external network forbids access to the intranet area, and the external network opens the corresponding www access port 80 to the server DMZ area.

The database access control list (ACL) specifies which type of personnel can access the database. For users who can access the database, access levels and roles determine the specific operations they can perform, such as creating or deleting documents, encrypting key sensitive information like encrypting passwords of users. In addition, the information source of the system is very diversified, so the information must be filtered to ensure that users can not publish bad information to the website. Secure Sockets Layer (SSL) is a security protocol which can protect data by encrypting the data when passing between the server and Web users, and SSL can be set on the server. And the unified control of the system resources, functions, users and roles could also strengthen the security of system privileges.

\section{Conclusion}

Video conference has the characteristics of integration, interaction and development of pictures, images and videos, which makes up for the drawbacks of traditional voice telephony. With the development of information technology in colleges and universities, more and more attention has been paid to the construction of cloud video conference platform. Cloud-based video conference will introduce new video technology into the meeting, interview, teaching and research, communication, evaluation, training and other fields.

\section{References}

[1] Shi Zuwei. Design and Implementation of Cloud Video Conference System in Colleges and Universities [J].Heilongjiang Science and Technology Information, 2014(10).

[2] Fu Kuo. Design and Implementation of Video Conference System [D]. Changchun: Jilin 
University, 2010.

[3] Du Lei, Xu Bo. Research on Cloud Video Conference Platform [J]. Microcomputer \& Its Applications, 2013(18).

[4] Qing Chen. Cloud and Video rise Quietly [J]. China new communications, 2012(4).

[5] Xie Bo, Cui Zhiqiang, Wang Zheng. Research on Integrated HD Video Conference Networking Based on Cloud Computing [J]. Television technology, 2014, 38(23).

[6] Dong He, Xu Lingyu. Software Service Flow Framework Based on Platforms [J]. Journal of Shanghai University: Natural Science Edition, 2013, 19(1):137-141.

[7] Guo Yong, Li Xiao, Huang Zhenyi, Lu Chunhua. Design and Implementation of Cloud Oriented Video Conference System [J]. Value engineering, 2014(23).

[8] Niu Xiaojing. Key Technologies of Cloud Media in Video Conferencing [J]. Theoretical research on urban construction, 2013(33). 